\title{
Anatomic Considerations of Intervertebral Disc Perspective in Lumbar Posterolateral Approach via Kambin’s Triangle: Cadaveric Study
}

\author{
Thamrong Lertudomphonwanit, Gun Keorochana, Chaiwat Kraiwattanapong, \\ Pongsthorn Chanplakorn, Pittavat Leelapattana, Wiwat Wajanavisit \\ Department of Orthopedics, Faculty of Medicine, Ramathibodi Hospital, Mahidol University, Bangkok, Thailand
}

\begin{abstract}
Study Design: Anatomical study.
Purpose: To evaluate the anatomy of intervertebral disc (IVD) area in the triangular working zone of the lumbar spine based on cadaveric measurements.

Overview of Literature: The posterolateral percutaneous approach to the lumbar spine has been widely used as a minimally invasive spinal surgery. However, to our knowledge, the actual perspective of disc boundaries and areas through posterolateral endoscopic approach are not well defined.

Methods: Ninety-six measurements for areas and dimensions of IVD in Kambin's triangle on bilateral sides of L1-S1 in 5 fresh human cadavers were studied.

Results: The trapezoidal IVD area (mean \pm standard deviation) for true working space was $63.65 \pm 14.70 \mathrm{~mm}^{2}$ at L1 $-2,70.79 \pm 21.88 \mathrm{~mm}^{2}$ at L2-3, 99.03 $\pm 15.83 \mathrm{~mm}^{2}$ at L3-4, 116.22 $\pm 20.93 \mathrm{~mm}^{2}$ at $L 4-5$, and $92.18 \pm 23.63 \mathrm{~mm}^{2}$ at L5-S1. The average dimension of calculated largest ellipsoidal cannula that could be placed in IVD area was $5.83 \times 11.02 \mathrm{~mm}$ at L1-2, $6.97 \times 10.78 \mathrm{~mm}$ at L2-3, 9.30×10.67 $\mathrm{mm}$ at $L 3-4,8.84 \times 13.15 \mathrm{~mm}$ at $L 4-5$, and $6.61 \times 14.07 \mathrm{~mm}$ at $L 5-S 1$.

Conclusions: The trapezoidal perspective of working zone of IVD in Kambin's triangle is important and limited. This should be taken into consideration when developing the tools and instruments for posterolateral endoscopic lumbar spine surgery.
\end{abstract}

Keywords: Working zone; Intervertebral disc; Posterolateral approach; Lumbar spine; Endoscopic approach

\section{Introduction}

Minimally invasive surgery has gained popularity in many fields of surgery, especially in the lumbar spinal region. In this area, the posterolateral percutaneous approach is commonly performed as Craig [1], Ottolenghi [2] and Valls et al. [3] have advocated it for the lumbar and lower thoracic spine since the 1950s.

In 1973, Kambin used central nucleotomy via a Craig cannula combined with laminectomy, and in 1975, Hijikata first introduced non-visualized posterolateral percutaneous nucleotomy via a 2.6-mm-outside diameter (od)cannula. In 1985, Onik et al. [4] used central nucleotomy shaver of $2.8 \mathrm{~mm}$ in diameter. Kambin began to use cannulas

Received Jan 19, 2016; Revised Mar 11, 2016; Accepted Apr 25, 2016

Corresponding author: Gun Keorochana

Department of Orthopedics, Faculty of Medicine, Ramathibodi Hospital, Mahidol University,

270 Rama VI Road, Ratchathewi, Bangkok 10400, Thailand

Tel: +66-2-201-1589, Fax: +66-2-201-1599, E-mail: gun_keo@yahoo.com 
with diameter 6.4-mm-od [5] and later introduced the concept of the unilateral biportal approach and oval cannulas [6] $(5 \times 8$ and $5 \times 10 \mathrm{~mm}$ inner diameter) that were designed to fit within a triangular working zone.

Kambin described this triangular working zone, in which the height of the zone is formed by the lateral border of the thecal sac corresponding to the medial pedicular border and extends from the superior endplate of the inferior vertebral body to superiorly located axillar of the exiting nerve root. The base of the triangle extends from the thecal sac to the medial aspect of the exiting nerve root. The exiting nerve root also defines the hypotenuse $[7,8]$.

Mirkovic et al. [7] defined the anatomic borders and dimensions of the safe zone and the largest safe working cannula diameter. The actual working zone is trapezoid shaped which correlates with the intervertebral disc (IVD) space area [9]. Nowadays, there are many minimally invasive procedures performed by accessing the IVD directly [10]. However, there is a risk of nerve root injury with this approach. Knowledge of the anatomy of IVD is very important and great care must be taken at the hypotenuse of the triangle. Current studies regarding the area of IVD in the triangular working zone are scarce, so the purpose of our study was to analyze the IVD area in the triangular working zone.

\section{Materials and Methods}

This study was approved by our Institutional Review Board. Five fresh human cadavers (average age at death 65 years; range 52-75 years; 3 males and 2 females), with no previous spinal surgery, were studied. The average height of the specimen was $165 \mathrm{~cm}$ (range, 154-178 cm). Specimens with age at death $<20$ years, gross deformity of lumbar spine, infection or tumor of the lumbar spine were excluded from the study. Cadavers were placed in the prone position with hips extended in lumbar lordosis.

Posterior soft tissues were removed from the underlying laminae of the vertebral arches from T12 to S1 bilaterally. The intertransverse ligament was removed to expose the extraforaminal region. A meticulous dissection was performed to preserve the anatomic relationship between bony, neural structures and IVD area. We also performed an osteotomy of the iliac crest to allow direct measurement of the L5-S1 level. Facetectomy for each level was performed to measure the nerve root length, the vertebral end plate, and the disc height. All measurements were performed bilaterally by caliper (precision $=0.1 \mathrm{~mm}$ ) by two authors and independently. Two intervertebral foramens from one specimen were excluded from our study due to sacralization of L5. A total of 96 measurements (48 intervertebral foramens) were studied.

The following measurements, in particular, were made: (1) the base of the triangular working zone at the superior end plate of the inferior vertebral body $(\mathrm{A})$, the distance between the lateral border of the thecal sac to medial aspect of the exiting nerve root; (2) the disc height (B), the distance between superior border and inferior border of IVD at the lateral border of dura; (3) the upper IVD distance (C), at the level of the superior border of IVD, the distance between the lateral border of dura and the intersection point of the corresponding nerve root and that IVD; (4) the dural height (D), the lateral border of the thecal sac extended from the superior end plate of the inferior vertebral body to superiorly located axillar of the exiting nerve root; and (5) the nerve root length (E), defines the hypotenuse of the triangular working zone, extended from the exiting site of the nerve root at lateral edge of dura to the intersection point of the nerve root and upper end plate of inferior vertebra. The methods of measurement are shown in Fig. 1.

Based on our data; a representative trapezoidal IVD area $(\mathrm{F})$, which we considered "the true working zone" for each lumbar level from L1-S1, was reconstructed and drawn in scale on graph paper. Then we calculated the IVD area of each level. We also defined the largest
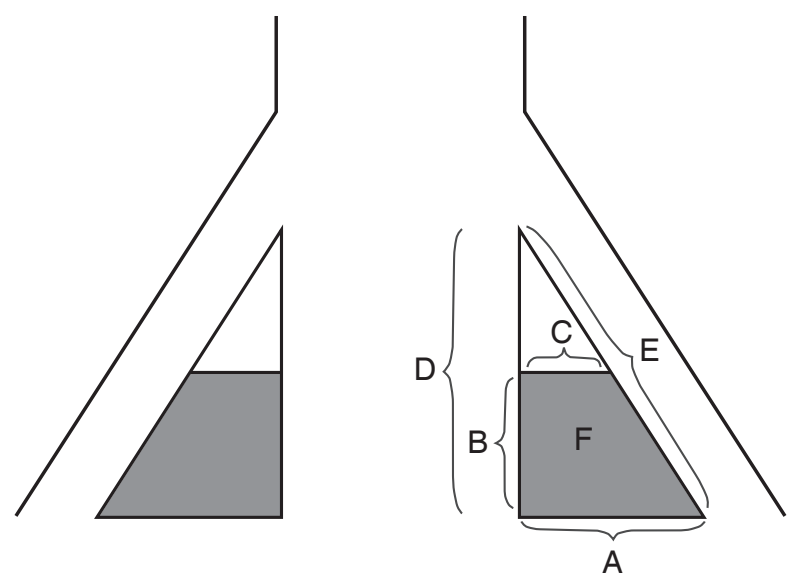

Fig. 1. Schematic diagram of the parameters of intervertebral disc (IVD) area and Kambin's triangle: $A$, base of triangle; $B$, disc height; $C$, upper disc distance; $D$, dural height; $E$, nerve root length; $F$, trapezoidal IVD area. 

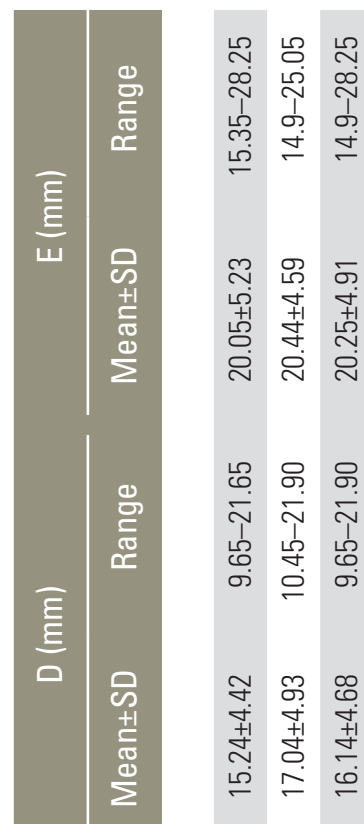

ֻ

ஸे

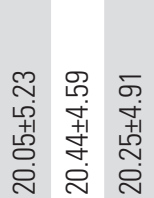

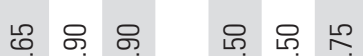

ஸे

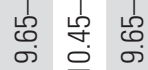

ปิ

ช. ๓ุ

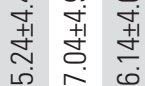

-

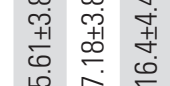

$\stackrel{\circ}{\circ}$

$\therefore 80$

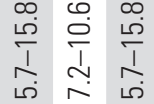

兽

인 농 농

市

ง.

르

흑

Ф

음

竞

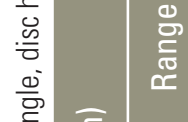

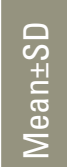

ㄷ.

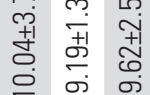

ช ำ

帒

는

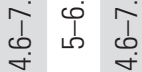

莽 ह

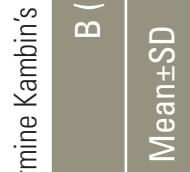

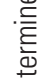

응

崩

袮

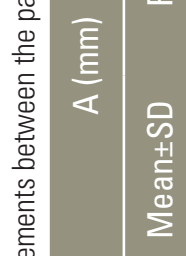

ल)

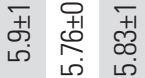

ก

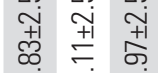

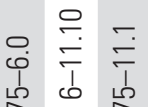

ㅇ. ช กิ

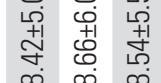

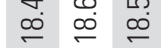

㐫

ฮั

ต ले

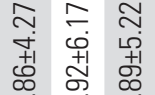

ฟ $~ ๗$

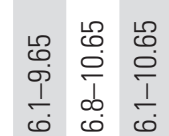

ำ

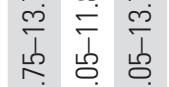

১

荿

ㅁ त

के का के

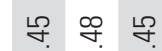

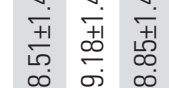

๓ m

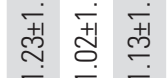

틀

ำ 아 웅

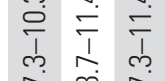

ก

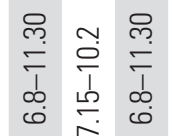

m 느

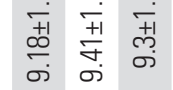

느 유 우

ฮั

० का का

๓. เே เ

m

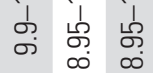

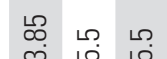

๓

ㅎํㅇ 잉

$\infty$

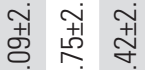

๗

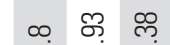

站 表 素

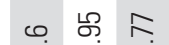

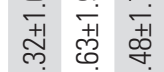

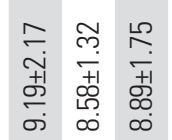

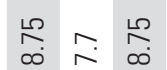

๗ே.

요

茾

เே ‡
-

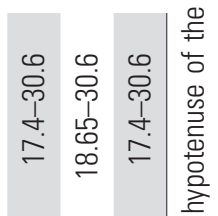

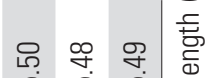

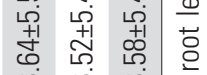

กั ฟิ ญ

노ำ 눈

六

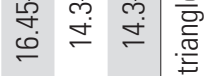

m

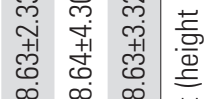

$\stackrel{\infty}{\circ} \stackrel{\infty}{\circ}$

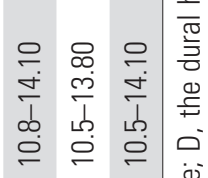

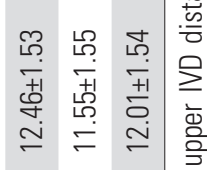

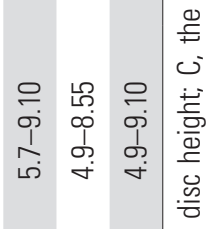

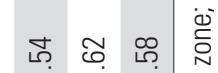

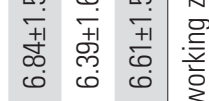

Б)

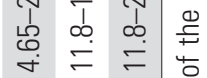

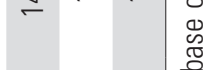

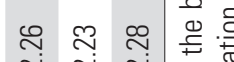

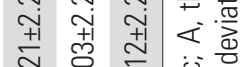

ํㅜㅇ

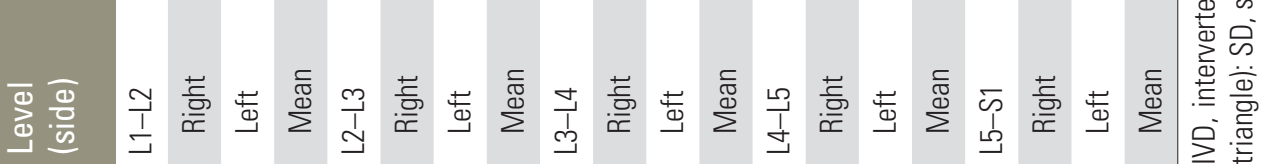


ellipsoidal working cannula that can be placed at IVD area for each lumbar level.

Mean and mean \pm standard deviation (SD) were used to describe the characteristics of the measurements. Paired $t$-test was used to compare right and left measurements. The mixed model was used to estimate and compare the differences between the lumbar levels. All the statistical analyses were performed using commercially available STATA ver. 13.

\section{Results}

The findings for all measurements are shown in Tables 1 and 2. The correlation of the parameters between lumbar levels is shown in Fig. 2.

\section{The base of the triangular working zone}

The distance of the base gradually increased from L1-2 to L5-S1 level, except at L2-3 level. The greatest length of the base was at L5-S1 level (mean, $16.12 \mathrm{~mm}$ ) and the narrowest distance of the base was at L2-3 level (mean, $12.04 \mathrm{~mm}$ ).

\section{Disc height}

The disc height gradually increased from L1-2 (mean, $5.83 \mathrm{~mm}$ ) to the greatest disc height at L3-4 level (mean, $9.30 \mathrm{~mm}$ ) and then decreased toward L5-S1 level (mean, $6.61 \mathrm{~mm})$.

Table 2. Area of IVD space and the largest oval cannula size

\begin{tabular}{lcrr} 
& \multicolumn{2}{c}{ Area of IVD $\left(\mathrm{mm}^{2}\right)$} & \\
\cline { 2 - 3 } Level & Mean (range) & Standard deviation & Cannula size (mm) \\
\cline { 2 - 3 } L1-L2 & $63.65(50.68-85.37)$ & 14.70 & $5.83 \times 11.02$ \\
L2-L3 & $70.79(47.43-99.37)$ & 21.88 & $6.97 \times 10.78$ \\
L3-L4 & $99.03(84.35-122.79)$ & 15.83 & $9.3 \times 10.67$ \\
L4-L5 & $116.22(90.03-146.84)$ & 20.93 & $8.84 \times 13.15$ \\
\hline L5-S1 & $92.18(70.29-124.77)$ & 23.63 & $6.61 \times 14.07$ \\
\hline
\end{tabular}

IVD, intervertebral disc.

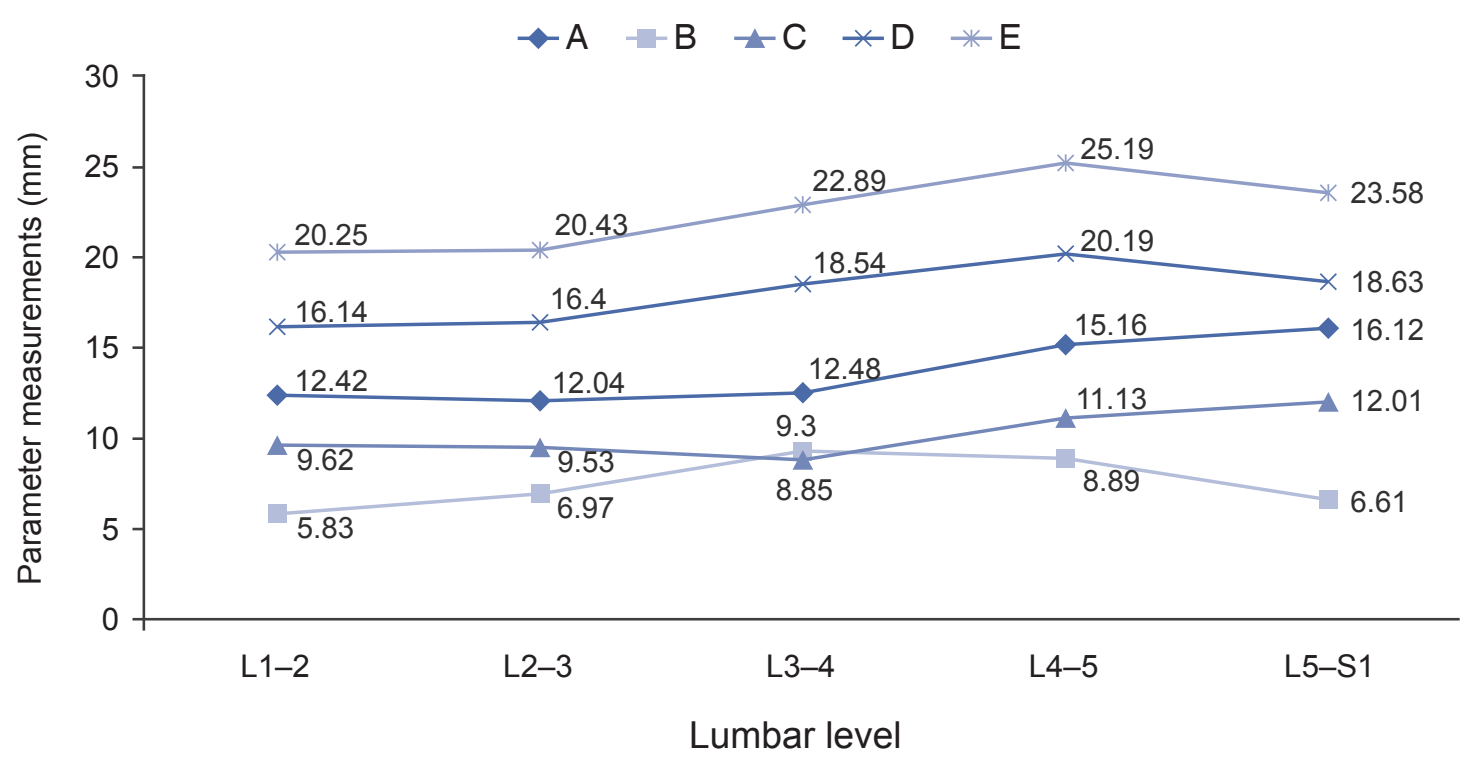

Fig. 2. Correlation of the parameters between lumbar levels: A, base of the triangular working zone; B, disc height; $C$, upper intervertebral disc distance; $D$, dural height; $E$, nerve root length. 


\section{Upper IVD distance}

The upper IVD distance extended from lateral border of dura to the intersection point of the corresponding nerve root and that IVD (Fig. 1). The upper IVD distance gradually decreased from L1-2 (mean, $9.62 \mathrm{~mm}$ ) to L3-4 (mean, $8.85 \mathrm{~mm}$ ) and then gradually increased toward L5-S1 (mean, $12.01 \mathrm{~mm}$ ). The greatest upper IVD distance was at L5-S1 (mean, $12.01 \mathrm{~mm}$ ) and the narrowest upper IVD distance was at $\mathrm{L} 3-4$ (mean, $8.85 \mathrm{~mm}$ ).

\section{Dural height (height of the triangle)}

The distance of the height of the triangle gradually increased from L1-2 to L4-5 foramina (mean, 16.14-20.19 $\mathrm{mm}$ ), then decreased toward L5-S1 (mean, $18.63 \mathrm{~mm}$ at L5-S1). The greatest distance of the height of the triangle was at L4-5 level (mean $20.19 \mathrm{~mm}$ ) and the narrowest distance was at L1-2 (mean, $16.14 \mathrm{~mm}$ ).

\section{Nerve root length (hypotenuse of the triangle)}

The distance of the hypotenuse of the triangle gradually increased from L1-2 (mean, $20.25 \mathrm{~mm}$ ) to L4-5 (mean, $25.19 \mathrm{~mm}$ ), while there was some decrease at L5-S1 level (mean, $23.58 \mathrm{~mm}$ ). The greatest distance of the hypotenuse was at L4-5 level (mean, $25.19 \mathrm{~mm}$ ) and the narrowest distance of the hypotenuse was at L1-2 level (mean, $20.25 \mathrm{~mm}$ ).

\section{IVD area of the actual working zone and the oval cannula size}

The actual working zone is trapezoidal in shape, which correlated with IVD area (Table 2). The IVD area of the actual working zone gradually increased from L1-2 (mean, $63.65 \mathrm{~mm}^{2}$ ) to L4-5 level (mean, $116.22 \mathrm{~mm}^{2}$ ), and then decreased toward L5-S1 (mean, $92.18 \mathrm{~mm}^{2}$ ).

The largest size of the oval cannula that could be placed at the actual working zone of the IVD area correlated with the disc height, the upper IVD distance, and the base of the triangular safe zone. The horizontal diameter was limited by the upper IVD distance and the base of the triangular safe zone; at the same time, the vertical diameter was limited by the disc height. The narrowest vertical diameter of the cannula size was at L1-2 (mean, $5.83 \mathrm{~mm}$; range, $5.83-6.61 \mathrm{~mm})$. The narrowest horizontal diameter of the cannula size was at L3-4 (mean, $10.6 \mathrm{~mm}$; range, $10.6-14.1 \mathrm{~mm})$.

\section{Discussion}

The percutaneous posterolateral approach to the spine has successfully been used in management of lumbar disc herniation since the 1970s [11]. However, many authors have expressed concern about the safety of the technique for the posterolateral percutaneous procedure. Yeung and Tsou [12] stated that safe and effective access was limited by the narrow channel, and there was little working space as compared with the procedure for knee and shoulder joints. Theoretically increased cannula diameters allow insertion of larger instruments to perform complex procedures but a large cannula diameter is directly related to nerve root injury [7].

Mirkovic et al. [7] stated that there were two issues of concern for endoscopic posterolateral approach. The first was the optimal circular cannula diameter and the second was the safe point of cannula insertion. However, neither of those concerns considered the anatomic dimensions of IVD in the Kambin's triangle. In our study, we believe that the true working area of percutaneous posterolateral approach through Kambin's triangle is trapezoidal in shape and correlated with IVD (in coronal plane). Min et al. [9] reported that the borders of the working zone, except the length of the base, may not be of clinical importance, which is contrary to our study's results. The largest size of cannula for posterolateral endoscope is limited by the parameters chosen in our study; these include not only the disc height and the base of the triangle, but also the upper IVD distance.

From previous cadaveric measurements, it was determined that cannula ranging from $4-10 \mathrm{~mm}$ could be safely used in the triangular safe zone $[7,8]$. One study reported that a $6.3-\mathrm{mm}$ cannula located in the midline of pedicle appears to be safe. A point of insertion at medial one third of the pedicle enables safe placement of a 7.5-mm cannula. Wimmer and Maurer [13] studied the anatomic considerations for percutaneous lumbar interbody fusion, and they reported that the maximum safe cannula diameter was $8 \mathrm{~mm}$ from L1-L4 and $7 \mathrm{~mm}$ from L4-S1. However, those studies reported only the safe margin of the circular cannula diameter. Our result demonstrated that the true working zone of posterolateral approach to IVD is trapezoidal in shape rather than the 
typical right-angle triangle. Use of a specially shaped endoscope that properly fits the true working area, such as with an oval or rectangular shape, might in fact increase the size of the working channel and the ability to use larger instruments. This may be appropriate and suitable for more advanced endoscopic procedures such as interbody cage fusion or nucleus replacement. The dimensions of the trapezoidal area from our data suggest that the limitation was mainly from upper IVD distance, of which the average length ranges from 8.85 to $9.62 \mathrm{~mm}$ at L1-4 and from $11.13-12.01 \mathrm{~mm}$ at $\mathrm{L} 4-\mathrm{S} 1$, which are larger than outer diameter of most of the current endoscopic systems. We also calculated the maximal oval size that can inserted into the true working trapezoidal area, which could guide us to the application of the proper size of oval cannula.

Furthermore, our study revealed the area of this trapezoidal working zone to range from 63.65 to $116.22 \mathrm{~mm}^{2}$, which is not mentioned in previous studies. Fortunately, it might be possible for this area to be enlarged. Disc height could be increased by disc distraction; the upper IVD distance and base of triangle might be widened by ability of nerve root traction. It is feasible to increase the working area in advanced endoscopic spine procedures. The actual cannula size could be larger compared with that in the anatomical study, if specially designed tools are developed, and allow enlarging this area, or if an expandable type of cannula is devised. Therefore, from our results, there is feasibility to develop larger and appropriate size of endoscopic system for more complex procedures by transforaminal approach such as interbody fusion, with new designed cages or use of larger instruments or special expandable implants.

It is important to realize that there are many other factors to consider in the posterolateral approach to the safe zone. First, there is the unique anatomical variation in the triangular safe zone. Osman and Marsolais [14] stated that the angular range for safely performed diskoscopies between T12 to L3 (38-60 degrees) is different from those of L3-S1 level (40-65 degrees). Arslan et al. [15] reported that the L1, L2, and L3 nerve root crossed the disc level closest to lateral border of foramen. Therefore these roots compared with lower level may be at a lower risk of injury during transforaminal percutaneous endoscopic discectomy. There is special consideration for L5-S1 transforaminal access. L5-S1 level might have a unique anatomical limitation such as a large facet joint, presence of ala, high iliac crest and narrow foramen. Second is degenerative change. Kim et al. [16] stated that hypertrophic facet joint with ligamentum flavum thickening may limit the actual dimensions of the safe working zone. Finally, regarding the visceral structures in this area, Baek and Lee [10] reported that extraforaminal ligaments, transforaminal ligaments and foraminal ligamentum flavum could cause tethering of the exiting nerve root and cause of an obstacle in operating on the exiting nerve root. Due to these problems, Kim et al. [16] suggested close monitoring and assessment of the pain response to inserted instruments and reviewing the imaging studies of the patients preoperatively to identify any congenital anomaly in the nerve root distorting the normal anatomy.

There were some limitations to our study. First, in this study we analyzed only two dimensions of the working zone, that is the coronal plane, with the facet joint removed, but the working zone is actually a three dimensional structure. Second, the diameter of oval cannula from our analysis is based on disc height, base of the triangular safe zone and the upper IVD. In fact, the value of the cannula diameter can be larger than the disc height, because the operator can insert the eccentric cannula within the safe zone to access to the disc. The cannula also helps to distract the disc space. Mirkovic et al. [7] and Kim et al. [16] have stated a confirmatory opinion about this. Third, we studied only Thai cadavers. Since the anatomy of spine in Asian population could be smaller than Caucasian or African people, this may be a limitation in a global application of our study. Lastly, our study did not include other factors that could affect the size of the cannula, such as angle of cannula insertion or the anatomic variation in each patient, for e.g., large hypertrophic facet joint degeneration or high iliac crest.

\section{Conclusions}

The trapezoidal perspective of the working zone of IVD in Kambin's triangle is important, yet limited. This should be taken into consideration when developing the tools and instruments for posterolateral endoscopic lumbar spine surgery.

\section{Conflict of Interest}

No potential conflict of interest relevant to this article was reported. 


\section{References}

1. Craig FS. Vertebral-body biopsy. J Bone Joint Surg Am 1956;38:93-102.

2. Ottolenghi CE. Diagnosis of orthopaedic lesions by aspiration biopsy: results of 1,061 punctures. J Bone Joint Surg Am 1955;37:443-64.

3. Valls J, Ottolenghi CE, Schajowicz F. Aspiration biopsy in diagnosis of lesions of vertebral bodies. J Am Med Assoc 1948;136:376-82.

4. Onik G, Helms CA, Ginsburg L, Hoaglund FT, Morris J. Percutaneous lumbar diskectomy using a new aspiration probe. AJR Am J Roentgenol 1985; 144:1137-40.

5. Kambin P, Gellman H. Percutaneous lateral discectomy of the lumbar spine: a preliminary report. Clin Orthop Relat Res 1983;174:127-32.

6. Kambin P. Diagnostic and therapeutic spinal arthroscopy. Neurosurg Clin N Am 1996;7:65-76.

7. Mirkovic SR, Schwartz DG, Glazier KD. Anatomic considerations in lumbar posterolateral percutaneous procedures. Spine (Phila Pa 1976) 1995;20:1965-71.

8. Kambin P, Brager MD. Percutaneous posterolateral discectomy: anatomy and mechanism. Clin Orthop Relat Res 1987;(223):145-54.

9. Min JH, Kang SH, Lee JB, Cho TH, Suh JK, Rhyu IJ. Morphometric analysis of the working zone for endoscopic lumbar discectomy. J Spinal Disord Tech 2005;18:132-5.

10. Baek OK, Lee SH. Extraforaminal lumbar interbody fusion for the treatment of isthmic spondylolisthesis. J Spinal Disord Tech 2009;22:219-27.

11. Kambin P. Arthroscopic and endoscopic spinal surgery. In: Kambin P, editor. Arthroscopic and endoscopic spinal surgery. Totowa: Humana Press; 2005. p.1-28.

12. Yeung AT, Tsou PM. Posterolateral endoscopic excision for lumbar disc herniation: Surgical technique, outcome, and complications in 307 consecutive cases. Spine (Phila Pa 1976) 2002;27:722-31.

13. Wimmer C, Maurer H. Anatomic consideration for lumbar percutaneous interbody fusion. Clin Orthop Relat Res 2000;(379):236-41.

14. Osman SG, Marsolais EB. Posterolateral arthroscopic discectomies of the thoracic and lumbar spine. Clin Orthop Relat Res 1994;(304):122-9.

15. Arslan M, Comert A, Acar HI, et al. Nerve root to lumbar disc relationships at the intervertebral foramen from a surgical viewpoint: an anatomical study. Clin Anat 2012;25:218-23.

16. Kim DH, Choi G, Lee SH. Endoscopic spine procedures. In: Kim DH, Choi G, Lee SH, editors. Endoscopic spine procedures. New York: Thieme; 2011. p.92-107. 